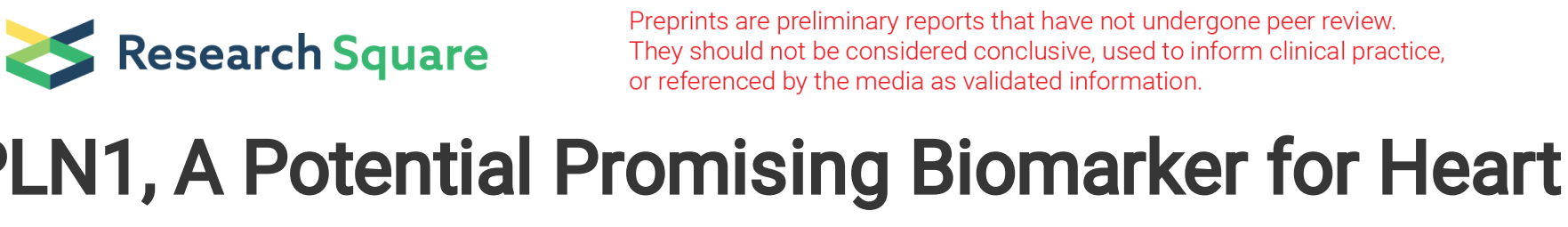 Failure
}

\section{Sichi Xu}

Chinese Academy of Medical Sciences and Peking Union Medical College

\section{Peipei Chen}

Chinese Academy of Medical Sciences and Peking Union Medical College

\section{Ying Xiao}

Chinese Academy of Medical Sciences and Peking Union Medical College

\section{Wei Wu}

Chinese Academy of Medical Sciences and Peking Union Medical College

Shuyang Zhang ( $\sim$ shuyangzhang103@nrdrs.org )

Chinese Academy of Medical Sciences and Peking Union Medical College

\section{Research Article}

Keywords: heart failure, biomarker, diagnostic

Posted Date: January 27th, 2022

DOI: https://doi.org/10.21203/rs.3.rs-1242640/v1

License: (c) (i) This work is licensed under a Creative Commons Attribution 4.0 International License. Read Full License 


\section{Abstract}

Objective: Heart failure (HF) is the end pathophysiological process of many cardiovascular diseases. Although brain natriuretic peptide (BNP) and N-terminal pro B type natriuretic peptide, (NT-proBNP) have been widely used to diagnose $\mathrm{HF}$, their expression levels are easily affected by age, weight, obesity, and renal function. Therefore, more accurate markers of HF are needed. This study aimed to explore common new biomarkers and mechanisms for HF based on microarray and bioinformatics analysis at the transcriptome level.

Methods: Microarray datasets of HF were downloaded from the Gene Expression Omnibus (GEO) database and differentially expressed genes (DEGs) were selected by using R software. Tissue or organspecific genes were predicted by BioGPS. Gene Ontology (GO) and Kyoto Encyclopedia of Genes and Genomes (KEGG) pathways were used to conduct enrichment analysis. Hub genes were selected by a protein-protein interaction (PPI) network, and competitive endogenous RNA (ceRNA) networks were constructed by Cytoscape. The HF model was induced by aortic banding (AB) surgery. Furthermore, effective DEGs were further validated using reverse transcription-quantitative polymerase chain reaction (RT-PCR) analysis.

Results: Three datasets were downloaded from the GEO. As many as 950 DEGs were recoded from GSE57338 and GSE141910, and 43 common DEGs were identified. DEGs were mostly enriched in extracellular matrix structural components, extracellular adhesion, oxygen binding and carrying, apoptosis, and phagosomes. Four hub genes were identified from the intersection of the PPI and BioGPS system. The diagnostic level of natriuretic peptide A (NPPA), haemoglobin subunit beta (HBB), and hyaluronan and proteoglycan link protein 1(HAPLN1) were validated by a GSE116250 dataset. SNHG16miR-23-3p/miR-103-5p/HAPLN1 and XIST-miR-23-3p/ miR-103-5p/HAPLN1 might regulate the disease progression of HF at the transcriptional level. Moreover, the mRNA levels of HAPLN1 and CD163 were also validated in an animal model of $\mathrm{HF}$.

Conclusions: HAPLN1 might be a new potential biomarker of HF and CD163 may participate in the progression of HF. SNHG16-miR-23-3p/ miR-103-5p / HAPLN1 and XIST-miR-23-3p/miR-103-5p/HAPLN1 may be involved in the transcriptional regulation during HF.

\section{Background}

Heart failure (HF) is the most common final pathophysiological process of many cardiovascular diseases, and it poses a serious threat to human life and health. Owing to the increased age of the population and the extended life expectancy coupled with advanced medical conditions, the number of patients with $\mathrm{HF}$ is expected to continuously accumulate and become the most common reason for hospitalization [1]. Once patients enter the development stage of HF, they will become irreversible and need long-term drug and device maintenance to slow down the development of the disease. Since there is no effective method to completely reverse HF at present, early detection and intervention into HF as early 
as possible to delay the progression of the disease are of great importance. Although many HF markers have been proposed, none of them could completely satisfy the need for both sensitivity and specificity. Indicators with high sensitivity are affected by many factors, such as brain natriuretic peptide (BNP) and $\mathrm{N}$-terminal pro-brain natriuretic peptide (NT-proBNP)[2]. Therefore, we need to explore and discover new biomarkers of HF. Transcription and microarray data analysis has been widely used to identify new biomarkers including both tumors and common diseases that rely on bioinformatic analysis. Moreover, emerging evidence has indicated a pivotal role of noncoding RNAs (ncRNAs) in remodeling related cardiovascular diseases as shown in transcriptome and chip technology experiment [3]. In addition, competitive endogenous RNA (ceRAN) networks can reveal new potential mechanisms that regulate disease development at the transcriptional regulatory level[4]. In this study, we conducted a transcriptional microarray data analysis combined with ceRNA network construction to explore new biomarkers of HF. Hypertension is the most common disease among many cardiovascular diseases, and HF caused by hypertension is one of the main causes of myocardial remodeling. Therefore, early screening markers of HF caused by hypertension are of great significance for the diagnosis and treatment of HF. Pressure overload-induced HF under hypertension or aortic valve stenosis is characterized by initial compensated cardiac hypertrophy and subsequent aggravation into decompensated HF which could serve as a proper animal model mimic of hypertension-induced HF. The target gene will be more convincing after the verification by this model.

\section{Methods}

\section{Microarray data download}

Representative microarray data sites of human left ventricle heart tissue with various reasons for HF were downloaded. The typical gene expression profiles GSE57338, GSE116250, and GSE141910 were downloaded from the GEO (https://www.ncbi.nlm.nih.gov/geo/) database, among which GSE116250 was regarded as the validation dataset. The gene expression profiles of GSE57338 contain different HF samples of cardiac tissue from 313 subjects, including 95 patients with ischemic heart disease and 82 patients with dilated cardiomyopathy as well as 136 samples with non-failing donors' heart. GSE116250 dataset was comprised of 37 dilated cardiomyopathy, 13 ischemic cardiomyopathy, and 14 non-failing donors' heart. GSE141910 included 166 non-failing donor hearts. and 200 failing hearts of with peripartum cardiomyopathy (PPCM), hypertrophic cardiomyopathy (HCM), and dilated cardiomyopathy (DCM) samples. The specific information was shown in Table1.

Table1. GEO database of heart failure profile dataset 


\begin{tabular}{|llll|}
\hline Organ & Dataset ID & Control & HF \\
\hline Left ventricle & GSE57338 & 136 & 177 \\
\hline Left ventricle & GSE141910 & 166 & 200 \\
\hline Left ventricle & GSE116250 & 14 & 50 \\
\hline
\end{tabular}

\section{Differentially expressed genes (DEGs) screening}

DEGs expression of GSE57338 and GSE141910 between the HF groups and the control group was analyzed by using the Limma package of R. The empty probe was removed after corresponding to the gene. Once a gene was associated with a different probe, the average values of the different probes were regarded as the final expression values. All DEGs expressions levels were log2 transformed to quantitative the normalized signal intensity. An adjusted $P$ value less than 0.05 and log fold-change I $(\mathrm{FC}) \mid>1$ were considered thresholds for DEGs screening.

\section{Functional enrichment analysis, PPI interaction, and tissue specificity analysis}

DEGs were analysed by Gene Ontology (GO), Kyoto Encyclopedia of Genes and Genomes (KEGG), and the REACTOME cluster method using the profiler $\mathrm{R}$ package. $\mathrm{GO}$ terms included the compromise of biological processes (BP), cellular components (CC), and molecular functions (MF). Protein interaction networks were constructed according to the STRING dataset, and the individual node genes were rejected. Genes specifically expressed in cardiovascular tissues or cells among the differential genes were listed according to the BioGPS database. The screening criteria were as follows: (1) the expression value of transcripts located in a single organ system was 10 times greater than the median of all of the other organs and tissues, (2) the expression of the second most abundant tissue did not exceed one-third of the highest tissue expression [5]. Four hub genes were obtained by taking the intersection of the differential genes screened by the STRING dataset and BioGPS.

\section{Construction of the ceRNA network}

Four online miRNA databases were adopted for the prediction of target miRNAs including TargetScanHuman (), micro-T-CDS(), miRWalk (http://mirwalk.umm.uni-heidelberg.de/), and miRDB (). The relationship of mRNA and miRNA predicted by at least two databases was recorded, and the coexpression network was constructed the in Cytoscape. The interactions among miRNA, IncRNA, and circRNA were constructed in starbase (https://starbase.sysu.edu.cn/), and the ceRNA network was constructed in Cytoscape.

\section{Anima model of HF}

Adult male-specific pathogen-free (SPF) grade C57BL/ 6 mice $(n=30,8-10$ weeks old, weighing 23.5-25.5 g) were obtained from the Institute of Laboratory Animal Science, Chinese Academy of Medical Sciences, Beijing, China. After one week of adaptive feeding, the mice underwent aortic banding (AB) surgery to mimic the pressure overload-induced HF model. After the mice were anesthetize with $3 \%$ pentobarbital 
sodium by intraperitoneal injection, the thoracic aorta was stripped and exposed under a microscope vision. Then, a $27 \mathrm{G}$ blunt needle was placed next to the aortic segment and tightly tied to it by 7-0 silk thread. The blunt needle was pulled out after ligation, leading to almost $70 \%$ contraction. Sham-operated mice underwent a similar procedure without ligation of the aorta. After eight weeks, the mice were euthanized by the use of an overdose of sodium pentobarbital ( $200 \mathrm{mg} / \mathrm{kg}$, intraperitoneal injection). All of the experimental procedures were approved by the Ethics Committee of Peking Union Medical College Hospital. All of the experimental operation were conformed to the Guidelines of the Care and Use of Laboratory Animals published by the United States National Institutes of Health.

\section{Echocardiographic measurement}

At 8 weeks after the operation, echocardiography parameters were collected to assess the change in the left ventricular (LV)structure and function in mice by inhalation of $1.5 \%$ isoflurane. Echocardiography was conducted with the MyLab 30CV system (Esaote SpA) with a $10 \mathrm{MHz}$ linear array ultrasound transducer. All of the two-dimensional images were acquired from the LV parasternal long axis and parasternal shortaxis near the papillary muscles. The left ventricular end-systolic diameter (LVESD) and end-diastolic diameter (LVEDD), LV ejection fraction (EF), and LV fractional shortening (FS) were recorded at the papillary level in M-mode with a sweep speed of $50 \mathrm{~mm} / \mathrm{s}$.

\section{Histological Analysis}

After the collection of echocardiographic parameters, the mice were immediately euthanized. Biomarkers of HF such as body weight (BW), heart and lung weight (HW, LW), and tibial length ( $T L$ ) were specifically recorded for each mouse. Hearts were immersed in $10 \% \mathrm{kcl}$ and fixed with $10 \%$ formalin. After 24 hours, the hearts were embedded in paraffin and cut into $5-\mu \mathrm{m}$ sections transversely. Haematoxylin-eosin (HE) and picrosirius red (PSR) staining were used for histological analysis of the cardiac cross-sectional area (CSA) and fibrosis level. After staining, a digital analysis system (Image-Pro Plus, version 6.0; Media Cybernetics, Bethesda, MD, USA) was adopted to evaluate the CSA of the myocytes and the percentage of collagen.

\section{Real-Time Polymerase Chain Reaction Analysis}

RNA was extracted from the left ventricle of frozen hearts via TRIzol (Invitrogen; Thermo Fisher Scientific, Inc. cDNA was synthesized from $1 \mu \mathrm{g}$ RNA from each group using oligo(dT) primers. Quantitative gene expression was conducted using the Light Cycler 480 SYBER Green Master Mix (Roche Diagnostics). Relative target gene expression of mRNA was normalized to the internal control of glyceraldehyde-3phosphate dehydrogenase (GAPDH). All details about the primers were presented in Table 2.

Table 2. Primers used in the study 


\begin{tabular}{|lll|}
\hline Gene names & Forward & Reverse \\
\hline GAPDH & TCATCAACGGGAAGCCCATC & CTCGTGGTTCACACCCATCA \\
\hline NPPA & ACCTGCTAGACCACCTGGAG & CCTTGGCTGTTATCTTCGGTACCGG \\
\hline CD163 & ATGGGTGGACACAGAATGGTT & CAGGAGCGTTAGTGACAGCAG \\
\hline HALPN1 & CCCCCGTCTACTTGTGGAAG & TCCTGAGCCAAATGCTGTAGG \\
\hline
\end{tabular}

\section{Statistics analysis}

The R software package ggpubr, ggplot2 and ROC were used to identify hub genes. The data were expressed as the mean \pm standard error. Student's t-test was used to detect the differences between the two groups. IBM SPSS Statistics25 (SPSS, Inc., Chicago, IL, USA) was used to analyse the data and receiver operating characteristic curve (ROC) curve.

\section{Results}

\section{Differential genes screening}

Two sets of gene expression data GSE57338 and GSE141910 were downloaded from the GEO database. A total of 48 different genes were obtained from the GSE57338 dataset, including 25 up-regulated genes and 23 down-regulated genes, and 902 differential genes were collected from the GSE141910 dataset composed of 622 up-regulated genes and 280 down-regulated genes. (Figure $1 \mathrm{~A}-\mathrm{F}$ ). The two datasets had 679 samples, so the selected genes were fully representative.

\section{Enrichment of differentially expressed genes by GO, KEGG pathway, and REACTOME analysis}

A total of 43 stable differentially expressed genes were obtained from the two datasets which were in accordance with up and down regulated patterns in the GSE57338 and GSE 141910 datasets (Figure 2A). After $\mathrm{GO}$ enrichment analysis of 43 genes, it was found that the function of BP mainly focused on the adhesion of extracellular or epidermal cells, myocardial hypertrophy and regulation, the bone morphogenetic protein (BMP) signal pathway, inflammatory response, and blood pressure regulation. The cell component of CC was mainly enriched in extracellular collagen, blood particles, and other molecular components. MF was mainly enriched in extracellular matrix structural components, oxygen binding, and endopeptidase activity. (Figure 2C-E) Through KEGG enrichment analysis, the genes were mainly enriched in gap junctions, apoptosis, phagosomes, the CGMP-PKG signaling pathway, and tight junctions (Figure. $2 \mathrm{~B}$ ), which was consistent with the $\mathrm{GO}$ enrichment analysis. For the reactome analysis, DEGs were mainly enriched in interferon signal interleukins, asparagine-N-linked glycosylation, neutrophil degranulation, and regulation of Toll-like receptor (TLR) by endogenous ligand (Figure 2F-G). 
The PPI network of DEGs was constructed according to the STRING database, and individual differential gene nodes were deleted. The PPI network contained 25 differentially expressed genes (Figure 3A). The tissue-specific analysis of the DEGs showed that 37 of the 43 genes were tissue-specific expression genes (Table 3 and Figure 3B), among which 6 genes were specifically expressed in cardiovascularrelated tissues or cells, natriuretic peptide A (NPPA), hemoglobin subunit beta (HBB), eukaryotic translation initiation factor 1A Y-linked (EIF1AY), serpin family A member 3 (Serpina3), hyaluronan and proteoglycan link protein 1(HAPLN1) and CD163 (Figure 3C).

Table 3. specific expressed genes identified by BioGPS

Tissue-specific differentially expressed genes

Non-tissue specific differentially expressed genes

MGST1, SLCO4A1, MXRA5, SERPINE1, SFRP4, OGN, ASPN, ECM2, SMOC2, IL1RL1, MYOT, LYVE1, IFI44L, AOX1, MNS1, PHLDA1, LUM, FCN3, CYP4B1, HAPLN1, VSIG4, FRZB, PI16, ANKRD2, RNASE2, METTL7B, NPPA, CD163, COL14A1, PLA2G2A, SERPINA3, MYH6, HBB囚EIF1AY, MME『SFRP1囚USP9Y

PDE5A, ADAMTS4, ADAMTS9, FNDC1, FREM1, HMGCS2

\section{Identification of hub genes and validation of important gene expression levels with independent datasets}

Four hub genes (NPPA, HBB, CD163, and HAPLN1) were obtained by the intersection of 25 genes constituting the PPI network and 6 genes specifically expressed in the cardiovascular system (Figure 3C) The main functions of the NPPA gene were related to muscular hypertrophy, myocardial adaptation, and blood pressure regulation. The main functions of the HBB and HAPLN1 genes were oxygen binding and regulation of the extracellular matrix. The four selected key genes of HF were all significantly differentially expressed in the validation dataset. The expression of the NPPA, HBB, and HAPLN1 genes in patients with $\mathrm{HF}$ was significantly up-regulated. The up-regulation range of the NPPA gene was the largest, $\log F C=3.907$, and CD163 was down-regulated in patients with HF, $\log F C=1.108$ (Figure 3D-G)

\section{Diagnostic efficacy analysis based on the four hub genes of HF}

Except for CD163, all the other three genes demonstrated good diagnostic results, and the diagnostic accuracy of the HBB gene was as high as $93.14 \%$. The area under the curves (AUCs) of NPPA and HAPLN1 were estimated to 0.8734 and 0.8414 , respectively, which demonstrates a similar diagnostic value between NPPA and HAPLN1. The AUC value of CD163 was 0.2286 , which may discriminate HF patients from healthy people (Figure 4A-D).

\section{ceRNA network analysis}

The ceRNA network was constructed for the four key genes of HF (the mRNA and miRNA interaction relationship was obtained based on the public database, as well as the miRNA-IncRNA and miRNA- 
circRNA interactions). A ceRNA network was constructed for the four selected four key genes. It was found that each gene was regulated by multiple miRNAs, which suggests a new direction for the further treatment of HF by regulating their upstream genes (Figure 4E-H). A previous study revealed that the long non-coding RNAs (IncRNAs) SNHG16 and XIST were significantly upregulated in mouse hypertrophic hearts and phenylephrine (PE)-stimulated cardiomyocytes [6, 7], therefore, we speculate that SNHG16miR-23-3p/ miR-103-5p / HAPLN1 and XIST -miR-23-3p/miR-103-5p/HAPLN1 might be potential RNA regulatory methods of HF.

\section{Construction of HF model in mice}

Eight weeks after $A B$ surgery, the mice had developed obvious HF. After AB surgery, the mice manifested an obvious cardiac pathological remodeling phenotype, with increased HW/BW, LW/BW, and HW/TL ratios (Figure $5 A-C$ ). $A B$ mice also exhibited deteriorated cardiac function compared with sham-operated mice by echocardiographic examination with LVEDd, LVESd, FS, and EF (Figure 5D-G,M). The average CSA after $A B$ surgery by $H \& E$ staining also confirmed the adverse pathological changes of $A B$ surgery on cardiac remodeling (Figure $5 \mathrm{H}$ and $\mathrm{N}$ ). Fibrosis was evaluated by picrosirius red staining and $\mathrm{AB}$ surgery had resulted in obvious cardiac interstitial fibrosis (Figure $5 \mathrm{I}$ and 0 ). Based on these results, the successful establishment of AB-induced HF in mice was confirmed.

\section{Detection of mRNA level of DEGs in a mouse model of HF}

As shown in Figure 5I-L, the mRNA level of ANP was significantly higher than that of the sham-operated group which proved the successful establishment of the HF model. The level of HAPLN1 was upregulated, and CD163 was down-regulated in the HF group under pressure-overload induced heart failure.

\section{Discussion}

$\mathrm{HF}$ is one of the most common end-stages of various cardiovascular diseases. With the aging of the population and the advancements in acute cardiovascular treatment, the incidence of chronic HF was increasing. Once the patient enters the stage of HF, it will continue to progress irreversibly, and mortality will increase significantly. HF patients need to take drugs or receive mechanical treatment for a long time, which will lead to serious economic and living burdens on society and individuals. Therefore, early and accurate identification of HF plays an essential role in slowing down the progression of heart deterioration. Plasma levels of BNP or pro-BNP have been widely used in the diagnosis of HF, however, the level is easily affected by multiple factors such as age, obesity, and kidney function. Although new biomarkers such a as soluble isoform of suppression of tumorigenicity 2(sST2), growth differentiation factor-15(GDF15), heart-type fatty acid-binding protein (H-FABP), and galectin-3 have been widely discussed in terms of the diagnosis of HF, none of them can achieve a perfect diagnosis with satisfactory sensitivity and specificity [8]. Thus, further study is required for the detection of early and accurate biomarkers of HF. 
Our study demonstrated 43 common DEGs from GEO datasets of GSE57338 and GSE 141910 from various HF and non-HF donor hearts. GO and KEGG pathways indicated that regulation of the extracellular matrix, oxygenation, and the degradation of hemoglobin, and macrophage phagocytosis were the major mechanisms involved in the process of HF. Four hub genes were screened out after the PPI network and were validated by another GEO dataset and subsequently by ROC curve analysis. We identified that NPPA, HBB and HAPLN1 were significantly upregulated in HF, and CD163 was downregulated. Moreover, NPPA, HBB, and HAPLN1 all achieved a good diagnosis value. In addition, we constructed mRNA-miRNA co-expression and ceRNA regulation networks and speculate that SNHG16miR-23-3p/ miR-103-5p/HAPLN1 and XIST-miR-23-3p/miR-103-5p/HAPLN1 might be involved in the RNA regulated pathway. Furthermore, the mRNA levels of HAPLN1 and CD163 were also validated in an animal model of HF.

NPPA, also known as atrial natriuretic peptide (ANP), is a peptide hormone synthesized and released by atrial myocytes. The major function of ANP is to relax vascular smooth muscle and promote renal watersodium excretion. Elevated levels of ANP are released into the blood under atrial wall stress such as excessive blood volume and pressure, changes in body position, and elevated central venous pressure [9] [10]. ANP has been proved to be a vital diagnostic and prognostic serum marker in congestive HF[10][11]. Mid-regional pro-atrial natriuretic peptide (MR-proANP) has acquired a sensitivity ranging from 0.95 to 0.97 and a negative predictive value ranging from 0.90 to 0.97 at a recommended threshold of 120 $\mathrm{pmol} / \mathrm{L}$ according to a systematic review and meta-analysis [11]. The selection of NPPA also validates the accuracy and credibility of our study. HBB is an important molecule for oxygen delivery and is one of the main globin genes in the blood of mature organisms. HBB, together with HBA determines the structure of the hemoglobin polypeptide chain [12]. Its abnormal structure often induces blood diseases such as thalassemia and sickle cell anemia and it affects the metabolic function of the human kidney and liver [13]. Cell-free hemoglobin (CFH) is increased in patients with pulmonary arterial hypertension (PAH) compared with healthy individuals, and is positively associated with mean $\mathrm{PAH}$ and pulmonary vascular resistance and inversely associated with the cardiac index in PAH patients [14]. After being released from RBCs into blood, hemoglobin could serve as a potential oxidant and vasoconstrictor associated with poor clinical outcomes. Previous studies have demonstrated that the level of CFH is elevated in patients with sepsis and is associated with poor outcomes, including an increased risk of acute kidney dysfunction, myocardial injury, and death $[15,16]$. Moreover, an early study also discovered that the mRNA level of HBB was elevated in sudden cardiac death patients [17]. In addition, anemia and iron deficiency are both common comorbidities that coexist in patients with HF. Both complications were closely associated with a poor clinical condition and worse outcomes neither together nor independently[18]. Hence, we speculate that hemolysis may occur in patients with HF, resulting in increased HBB release. HAPLN1, which act as the combination platform of proteoglycan with hyaluronic acid has been reported to play a significant role in cell adhesion and extracellular matrix structure conformation [19]. HAPLN1 stabilizes interactions between versican and hyaluronan, and participates in the proliferation of fibroblasts and their conversion to myofibroblasts in cultured human lung fibroblasts [20].Previous studies have demonstrated that the cancer-associated fibroblasts (CAF) derived HAPLN1 is up-regulated in CAFs of gastric cancer and that 
increased HAPLN1 levels are accompanied by shorter overall survival. They also discovered that the upregulation of HAPLN1 was regulated by the activation of the transforming growth factor- $\beta$ (TGF- $\beta 1$ ) /Smad2/3 signaling pathway [21]. HAPLN1 was also related to the process of endocardial epithelial-tomesenchymal transformation (EMT) in valvular interstitial cells and deletion of HAPLN1 in mice resulted in hypoplastic valves during heart development which may imply a potential role for promoting EMT [22].Regarding the critical role of EMT and TGF- $\beta 1 / \mathrm{Smad} 2 / 3$ in the pathology of cardiac fibrosis, we speculate that HAPLN1 may participate in the development of cardiac fibrosis in HF. CD163 is a transmembrane glycoprotein specifically limited to the membrane surface of monocytes/macrophages, and it is defined as hemoglobin scavenger receptor (SRCR). Dynamic alteration of CD163 is considered to be one of major signs of macrophage switching to alternatively activated phenotypes under inflammation [23]. Pevious studies have illustrated that the serum level of CD163 is upregulated in patients with atrial fibrillation, coronary heart disease, and doxorubicin-related cardiac damage [24, 25]. The inflammatory response and macrophage infiltration mediate the main mechanism of HF and upregulated soluble decoy receptor CD163 (sCD163) was discovered in HF patients compared with healthy subjects by ELISA kits according to a previous study [26]. However, our study found a reduced level of CD163 in cardiac tissues which may deserve further study. An elevated level of HBB may consume CD163 positive macrophages which may result in reduced CD163.

Regarding the regulatory mechanism of RNA, LncRNAs play a significant role in the development of cardiac remolding mainly through the ceRNA mechanism [27].

MicroRNAs have been identified in tissue and are also involved in several pathophysiological processes related to HF, such as cardiac fibrosis and hypertrophy [28]. MicroRNAs can also be detected in the circulation, and are potential diagnostic and prognostic biomarkers in HF.

miR-23-3p was able to activate the TGF- $ß$ signal in human keratinocytes by increasing SMAD2 phosphorylation [29], moreover, miR-23-3p was also up-regulated in patients with liver cirrhosis compared with hepatocellular carcinoma (HCC) [30], which may indicate that miR-23-3p participates in the process of liver fibrosis. Another report also demonstrated that LINC00909 could promote the EMT transition of ovarian cancer cells via regulation of MRC2 by the function of ceRNA and sponging miR-23-3p[31]. All of these results indicated that miR-23-3p might participate in the regulation of the fibrosis process. miR-103$5 p$ was reduced in obese patients which indicates that miR-103-5p participates in lipid metabolism which was also associated with HF [32].

A previous study revealed that SNHG16 was upregulated in the cardiac hypertrophy model and downregulation of SNHG16 could inhibit the expression of hypertrophic markers and reduce CSA induced by Ang II [6]. Moreover, SNHG16 might also be involved in TGF- $\beta$ signaling and extracellular matrix (ECM)receptor interaction [33]. LncRNA XIST was increased in patients with chronic HF and played a regulatory role in cardiomyocyte function, moreover, XIST was also proven to be significantly upregulated in hypertrophic mouse hearts and PE-stimulated cardiomyocytes while knockdown of XIST alleviated PEinduced cardiomyocyte hypertrophy [34]. Therefore, we speculate that SNHG16-miR-23-3p/miR-103- 
5p/HAPLN1 and XIST -miR-23-3p/ miR-103-5p/HAPLN1 may participate in the development of HF and are probably involved in cardiac fibrosis. Given the differences in species, further prospective cohort studies and basic experiments are needed to confirm our views.

\section{Conclusions}

This study found that levels of HBB, HPLN1, ANP, and CD163 in HF hearts were different from those in control hearts, and proposed that HBB, and HPLN1 may be potential markers for the diagnosis of HF. The pressure load myocardial hypertrophy model also demonstrated obvious changes in CD163 and HPLN1. This study proposed for the first time that HPLN1 was involved in the process of HF, and SNHG16-miR-233p/miR-103-5p/HAPLN1 and XIST -miR-23-3p/miR-103-5p/HPLN1 may regulate HF development.

\section{Abbreviations}

HF Heart failure

BNP Brain natriuretic peptide

NT-proBNP $\quad \mathrm{N}$ terminal pro B type natriuretic peptide

GEO Gene Expression Omnibus

DEGs Differentially expressed genes

GO Gene Ontology

KEGG Kyoto Encyclopedia of Genes and Genomes

PPI Protein-protein interaction

ceRNA Competitive endogenous RNA

$A B \quad$ Aortic banding

RT-PCR Reverse transcription-quantitative polymerase chain reaction

NPPA Natriuretic peptide A

HBB Hemoglobin subunit beta

HAPLN1 Hyaluronan and proteoglycan link protein 1

ncRNAs Non-coding RNAs

PPCM Peripartum cardiomyopathy 
HCM Hypertrophic cardiomyopathy

DCM Dilated cardiomyopathy

BP Biological processes

CC Cellular components

MF Molecular functions

LV Left ventricular

EF Ejection fraction

FS Fractional shortening

BW Body weight

LW Lung weight

HW Heart weight

TL Tibial length

HE Haematoxylin-eosin

PSR Picrosirius red

CSA Cross-sectional area

GAPDH Glyceraldehyde-3-phosphate dehydrogenase

BMP Bone morphogenetic protein

TLR Toll-like receptor

EIF1AY Eukaryotic translation initiation factor 1A Y-linked

Serpina3 Serpin family A member 3

AUC Area under the curve

IncRNA Long non-coding RNA

PE Phenylephrine

sST2 Soluble isoform of suppression of tumorigenicity 2 
GDF15 Growth differentiation factor-15

H-FABP Heart-type fatty acid-binding protein

ANP Atrial natriuretic peptide

MR-proANP Pro-atrial natriuretic peptide

CFH Cell-free hemoglobin

CAFs Cancer-associated fibroblasts

TGF- $\beta 1$ Transforming growth factor- $\beta$

SRCR Scavenger receptor

sCD163 Soluble decoy receptor CD163

ECM Extracellular matrix

\section{Declarations}

\section{Acknowledgements}

Not applicable.

\section{Author Contributions}

SCX, WW and SYZ designed the research, SCX and PPC collected the data and conducted further enrichment analysis. YX analyzed the data and proved the last consequence. SCX wrote the primary manuscript, WW and SYZ wrote and revised the final manuscript. All of the authors listed have read and approved it for publication.

\section{Funding}

None.

\section{Availability of data and materials}

The gene expression profiles of GSE57338, GSE116250 and GSE141910 were downloaded from Gene Expression Omnibus (GEO). Other data used and analyzed in this study are available from the corresponding author on reasonable request.

\section{Ethics approval and consent to participate}


All methods were carried out in accordance with relevant guidelines and regulations. All the experimental procedures were approved by the Ethics Committee of Peking Union Medical College Hospital. All of the experimental operation were conformed to the Guidelines of the Care and Use of Laboratory Animals published by the United States National Institutes of Health. The study on animals was carried out in compliance with the ARRIVE guidelines. All animal experiments abided by the Regulations on the Management of Laboratory Animals and related ethic requirements.

\section{Consent for publication}

Not applicable

\section{Competing interests}

The authors declared no conflicts of interest regarding this work.

\section{References}

1. Ziaeian B, Fonarow GC: Epidemiology and aetiology of heart failure. Nature reviews Cardiology 2016, 13(6):368-378.

2. Francis GS, Felker GM, Tang WH: A Test in Context: Critical Evaluation of Natriuretic Peptide Testing in Heart Failure. Journal of the American College of Cardiology 2016, 67(3):330-337.

3. Zhou H, Wang B, Yang YX, Jia QJ, Zhang A, Qi ZW, Zhang JP: Long Noncoding RNAs in Pathological Cardiac Remodeling: A Review of the Update Literature. BioMed research international 2019, 2019:7159592.

4. Zheng Y, Zhang Y, Zhang X, Dang Y, Cheng Y, Hua W, Teng M, Wang S, Lu X: Novel IncRNA-miRNAmRNA Competing Endogenous RNA Triple Networks Associated Programmed Cell Death in Heart Failure. Frontiers in cardiovascular medicine 2021, 8:747449.

5. Wu H, Su QX, Zhang ZY, Zhang Z, Gao SL, Lu C, Zuo L, Zhang LF: Exploration of the core genes in ulcerative interstitial cystitis/bladder pain syndrome. International braz j urol: official journal of the Brazilian Society of Urology 2021, 47(4):843-855.

6. Wang D, Lin B, Zhang W, Wang X: Up-regulation of SNHG16 induced by CTCF accelerates cardiac hypertrophy by targeting miR-182-5p/IGF1 axis. Cell biology international 2020, 44(7):1426-1435.

7. Xiao L, Gu Y, Sun Y, Chen J, Wang X, Zhang Y, Gao L, Li L: The long noncoding RNA XIST regulates cardiac hypertrophy by targeting miR-101. Journal of cellular physiology 2019, 234(8):1368013692.

8. Biasucci LM, Maino A, Grimaldi MC, Cappannoli L, Aspromonte N: Novel Biomarkers in Heart Failure: New Insight in Pathophysiology and Clinical Perspective. Journal of clinical medicine 2021, 10(13).

9. Nakagawa Y, Nishikimi T, Kuwahara K: Atrial and brain natriuretic peptides: Hormones secreted from the heart. Peptides 2019, 111:18-25. 
10. Brandt RR, Wright RS, Redfield MM, Burnett JC, Jr.: Atrial natriuretic peptide in heart failure. Journal of the American College of Cardiology 1993, 22(4 Suppl A):86a-92a.

11. Roberts E, Ludman AJ, Dworzynski K, Al-Mohammad A, Cowie MR, McMurray JJ, Mant J: The diagnostic accuracy of the natriuretic peptides in heart failure: systematic review and diagnostic meta-analysis in the acute care setting. BMJ (Clinical research ed) 2015, 350:h910.

12. Williams TN: Red blood cell defects and malaria. Molecular and biochemical parasitology 2006, 149(2):121-127.

13. Zeng H, Liu X, Zhang Y: Identification of Potential Biomarkers and Immune Infiltration Characteristics in Idiopathic Pulmonary Arterial Hypertension Using Bioinformatics Analysis. Frontiers in cardiovascular medicine 2021, 8:624714.

14. Brittain EL, Janz DR, Austin ED, Bastarache JA, Wheeler LA, Ware LB, Hemnes AR: Elevation of plasma cell-free hemoglobin in pulmonary arterial hypertension. Chest 2014, 146(6):1478-1485.

15. Billings FTt, Ball SK, Roberts LJ, 2nd, Pretorius M: Postoperative acute kidney injury is associated with hemoglobinemia and an enhanced oxidative stress response. Free radical biology \& medicine 2011, 50(11):1480-1487.

16. Natanson C, Kern SJ, Lurie P, Banks SM, Wolfe SM: Cell-free hemoglobin-based blood substitutes and risk of myocardial infarction and death: a meta-analysis. Jama 2008, 299(19):2304-2312.

17. Son GH, Park SH, Kim Y, Kim JY, Kim JW, Chung S, Kim YH, Kim H, Hwang JJ, Seo JS: Postmortem mRNA expression patterns in left ventricular myocardial tissues and their implications for forensic diagnosis of sudden cardiac death. Molecules and cells 2014, 37(3):241-247.

18. Sharma YP, Kaur N, Kasinadhuni G, Batta A, Chhabra P, Verma S, Panda P: Anemia in heart failure: still an unsolved enigma. The Egyptian heart journal: (EHJ) : official bulletin of the Egyptian Society of Cardiology 2021, 73(1):75.

19. Nojima K, Miyazaki H, Hori T, Vargova L, Oohashi T: Assessment of Possible Contributions of Hyaluronan and Proteoglycan Binding Link Protein 4 to Differential Perineuronal Net Formation at the Calyx of Held. Frontiers in cell and developmental biology 2021, 9:730550.

20. Evanko SP, Gooden MD, Kang I, Chan CK, Vernon RB, Wight TN: A Role for HAPLN1 During Phenotypic Modulation of Human Lung Fibroblasts In Vitro. The journal of histochemistry and cytochemistry: official journal of the Histochemistry Society 2020, 68(11):797-811.

21. Zhang T, Li X, He Y, Wang Y, Shen J, Wang S, You Q, Zhai J, Shen L: Cancer-associated fibroblastsderived HAPLN1 promotes tumour invasion through extracellular matrix remodeling in gastric cancer. Gastric cancer: official journal of the International Gastric Cancer Association and the Japanese Gastric Cancer Association 2021.

22. DeLaughter DM, Christodoulou DC, Robinson JY, Seidman CE, Baldwin HS, Seidman JG, Barnett JV: Spatial transcriptional profile of the chick and mouse endocardial cushions identify novel regulators of endocardial EMT in vitro. Journal of molecular and cellular cardiology 2013, 59:196-204.

23. Wan GX, Ji LH, Xia WB, Cheng L, Zhang YG: Bioinformatics identification of potential candidate blood indicators for doxorubicin-induced heart failure. Experimental and therapeutic medicine 2018, 
16(3):2534-2544.

24. Zhong SM, Qin YH, Li ZC, Wei YS: [Clinical value of detecting serum soluble CD163 level in patients with atrial fibrillation]. Nan fang yi ke da xue xue bao = Journal of Southern Medical University 2016, 36(10):1406-1409.

25. Zou LY, Peng CQ, Li CZ, Zhao CL, Zhu JM, Liu JL, Zhang CX: [Association between hemoglobin scavenger receptor CD163 expression and coronary atherosclerotic severity in patients with coronary heart disease]. Zhonghua xin xue guan bing za zhi 2009, 37(7):605-609.

26. Ptaszynska-Kopczynska K, Marcinkiewicz-Siemion M, Lisowska A, Waszkiewicz E, Witkowski M, Jasiewicz M, Miklasz P, Jakim P, Galar B, Musial WJ et al: Alterations of soluble TWEAK and CD163 concentrations in patients with chronic heart failure. Cytokine 2016, 80:7-12.

27. Xie L, Zhang Q, Mao J, Zhang J, Li L: The Roles of IncRNA in Myocardial Infarction: Molecular Mechanisms, Diagnosis Biomarkers, and Therapeutic Perspectives. Frontiers in cell and developmental biology 2021, 9:680713.

28. Vegter EL, van der Meer P, de Windt LJ, Pinto YM, Voors AA: MicroRNAs in heart failure: from biomarker to target for therapy. European journal of heart failure 2016, 18(5):457-468.

29. Barbollat-Boutrand L, Joly-Tonetti N, Dos Santos M, Metral E, Boher A, Masse I, Berthier-Vergnes O, Bertolino P, Damour O, Lamartine J: MicroRNA-23b-3p regulates human keratinocyte differentiation through repression of TGIF1 and activation of the TGF-ß-SMAD2 signalling pathway. Experimental dermatology 2017, 26(1):51-57.

30. Elemeery MN, Mohamed MA, Madkour MA, Shamseya MM, Issa NM, Badr AN, Ghareeb DA, Pan CH: MicroRNA signature in patients with hepatocellular carcinoma associated with type 2 diabetes. World journal of gastroenterology 2019, 25(42):6322-6341.

31. Yang X, Wu G, Yang F, He L, Xie X, Li L, Yang L, Ma Y, Zhang Q, Chen J et al: Elevated LINC00909 Promotes Tumor Progression of Ovarian Cancer via Regulating the miR-23b-3p/MRC2 Axis. Oxidative medicine and cellular longevity 2021, 2021:5574130.

32. Villard A, Marchand L, Thivolet C, Rome S: Diagnostic Value of Cell-free Circulating MicroRNAs for Obesity and Type 2 Diabetes: A Meta-analysis. Journal of molecular biomarkers \& diagnosis 2015, $6(6)$.

33. Shi X, Shao X, Liu B, Lv M, Pandey P, Guo C, Zhang R, Zhang Y: Genome-wide screening of functional long noncoding RNAs in the epicardial adipose tissues of atrial fibrillation. Biochimica et biophysica acta Molecular basis of disease 2020, 1866(7):165757.

34. Sun ZL, Chen MH, Guo YN, Liu ZQ: LncRNA XIST is elevated in patients with chronic heart failure and has a regulatory role in cardiomyocyte function. Journal of biological regulators and homeostatic agents 2021, 35(2):677-682.

\section{Figures}


A

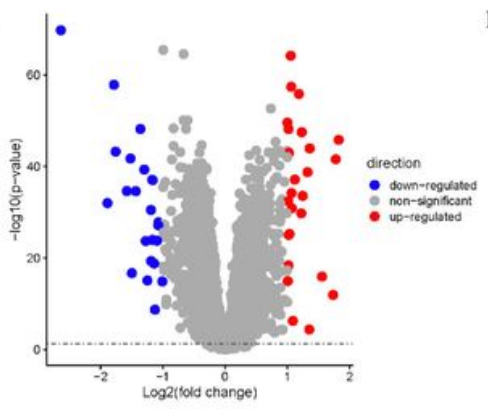

D

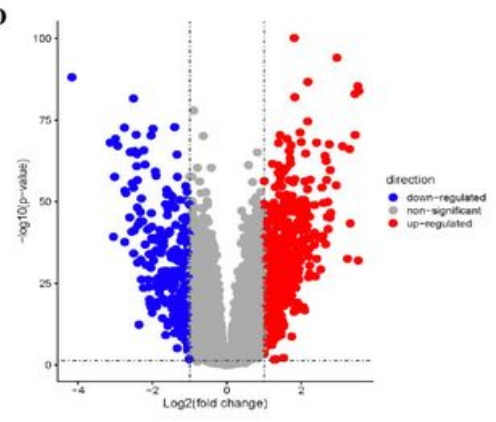

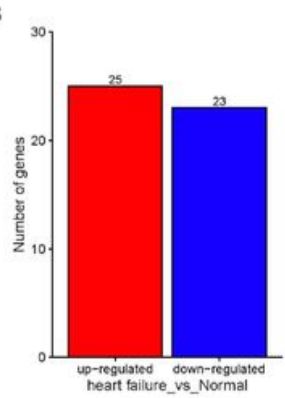

E

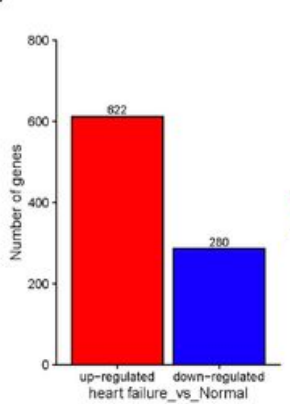

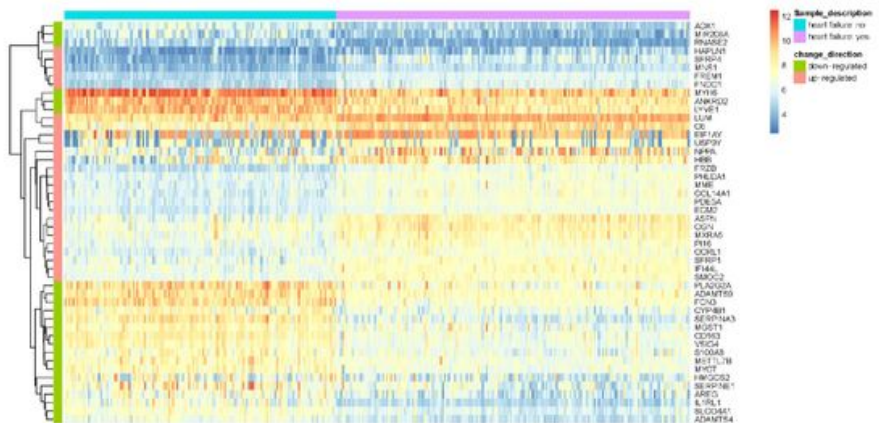

F

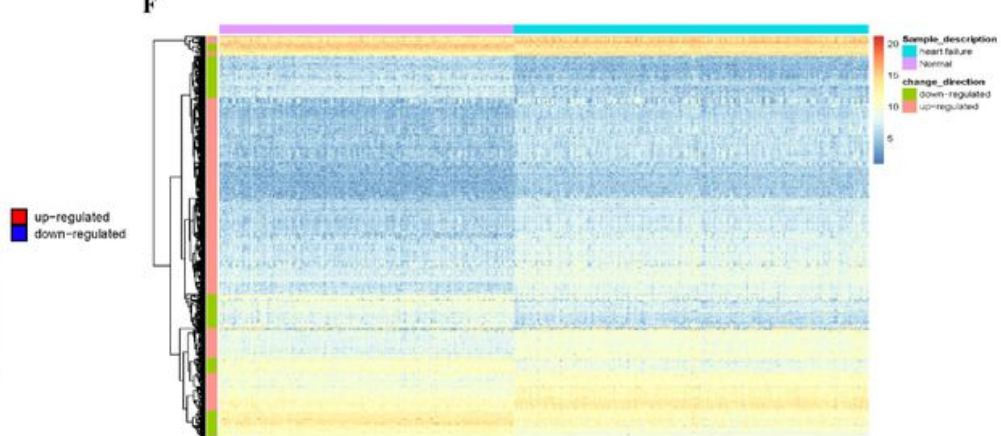

\section{Figure 1}

Differential genes in the GSE57338 and GSE141910 data sets. Fig. note: GSE57338: A-differential gene volcano map; B- quantitative distribution of up and down-regulated genes; C-differential gene expression profile; GSE141910: D-differential gene volcano map; E- quantitative distribution of up and downregulated genes; F-differential gene expression profile 

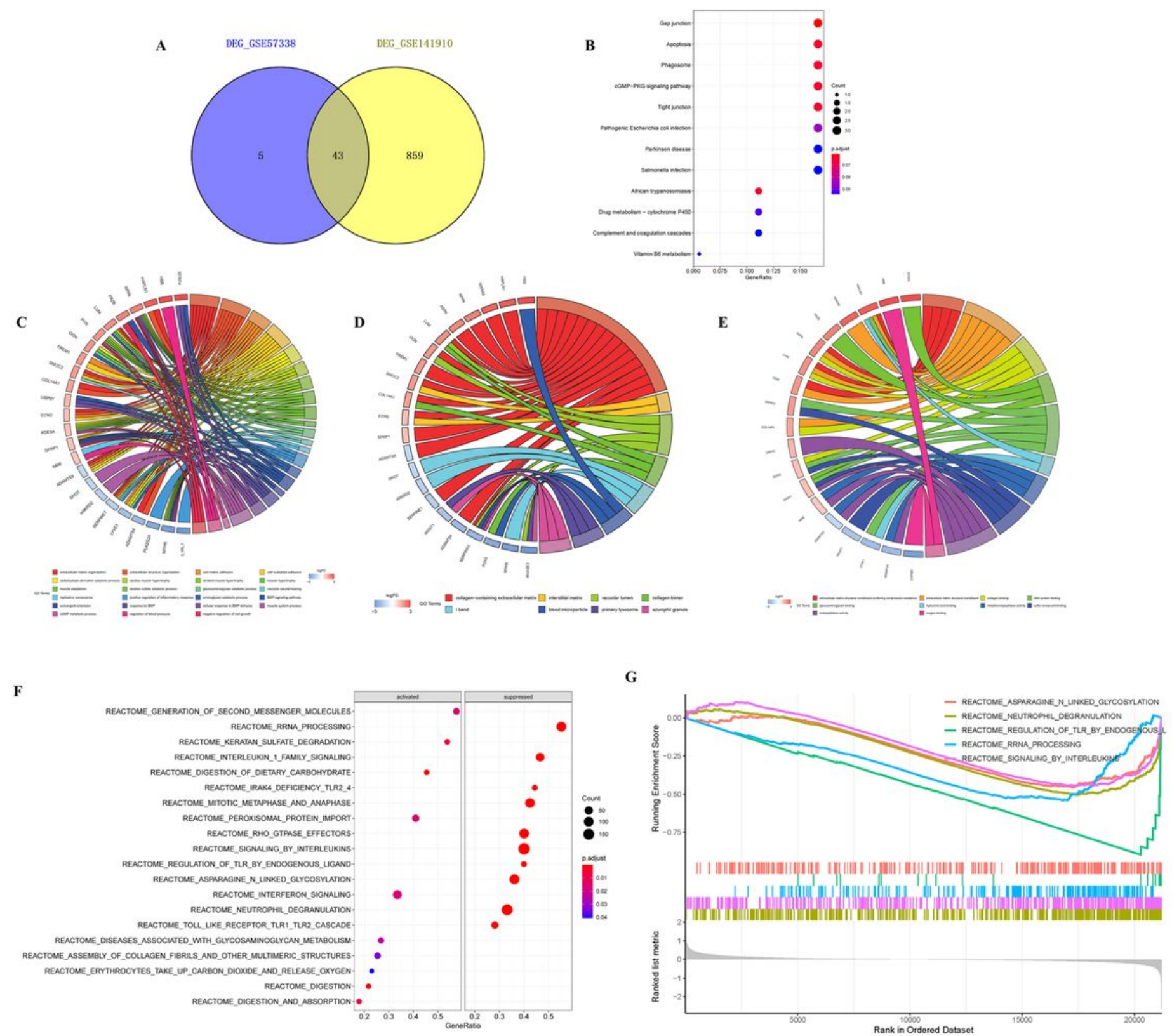

\section{Figure 2}

Gene enrichment analysis. Fig. note: A: The vein map of common genes between GSE57338 and GSE 141910 data sets; B: KEGG enrichment analysis; C: GO enrichment analysis of BP; D: GO enrichment analysis of CC; F: GO enrichment analysis of MF; F: Reactome enrichment plot; G: GSEA analysis for the first five specific pathways of Reactome enrichment 

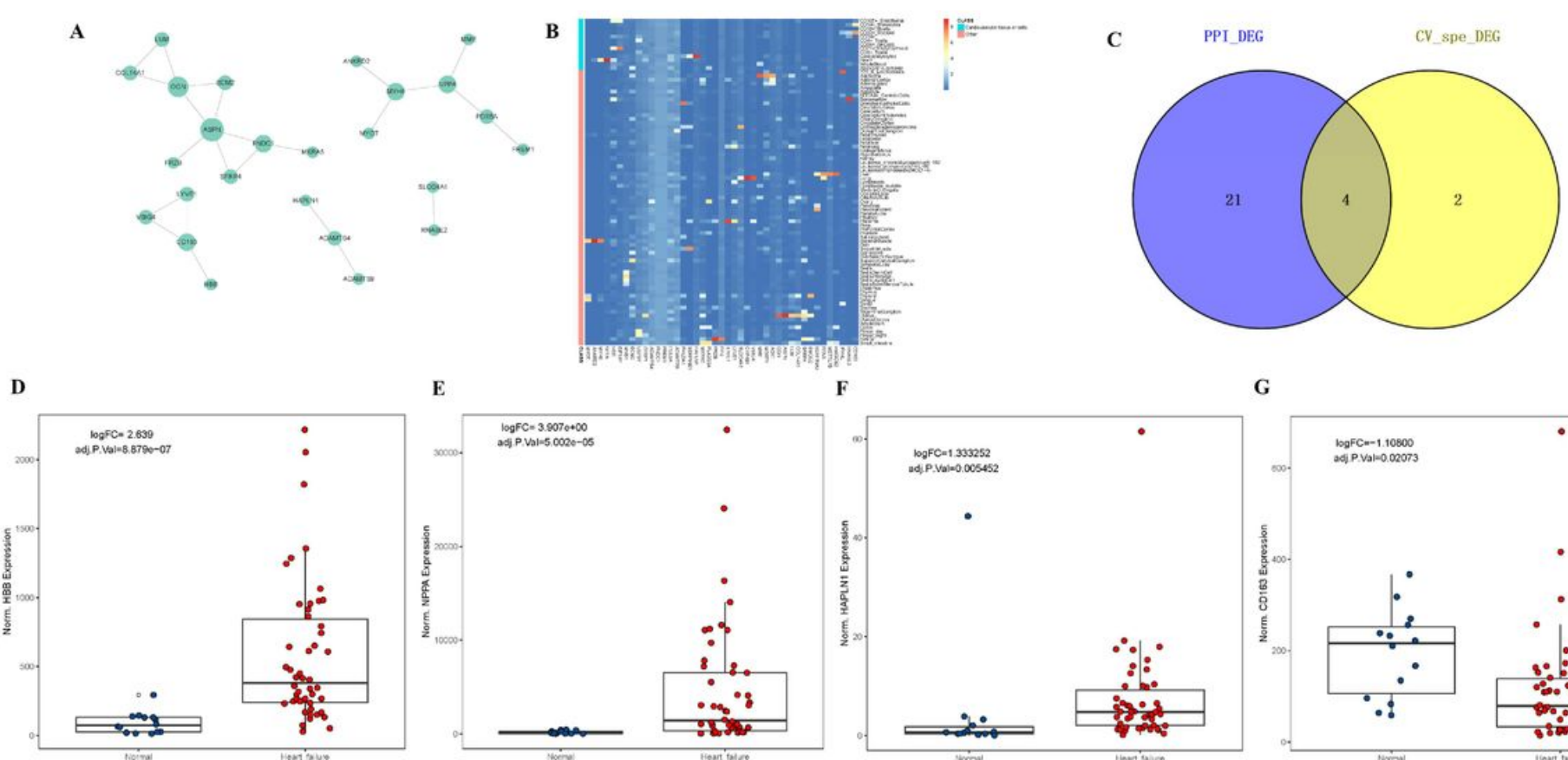

F

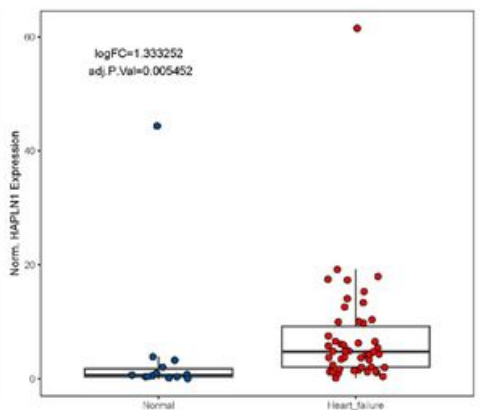

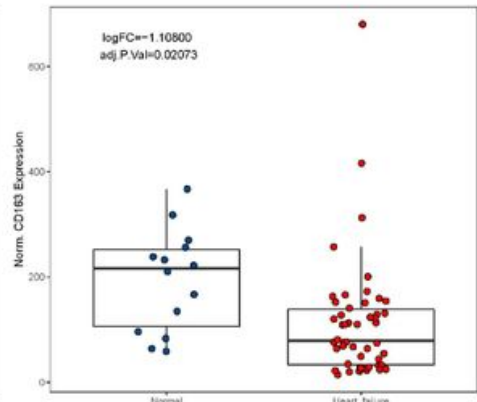

Figure 3

The identification of hub gene and the expression values. A: Construction of PPI network by DEGs. B: Tissue expression profiles of different genes in BioGPS C: Different genes in PPI network and vein map specifically expressed in the cardiovascular system; D: Independent validation of HBB gene in GSE116250 data set; E: Independent validation of NPPA gene in GSE116250 data set; F: Independent validation of Hapln1 gene; G: Independent validation of --CD163 gene.
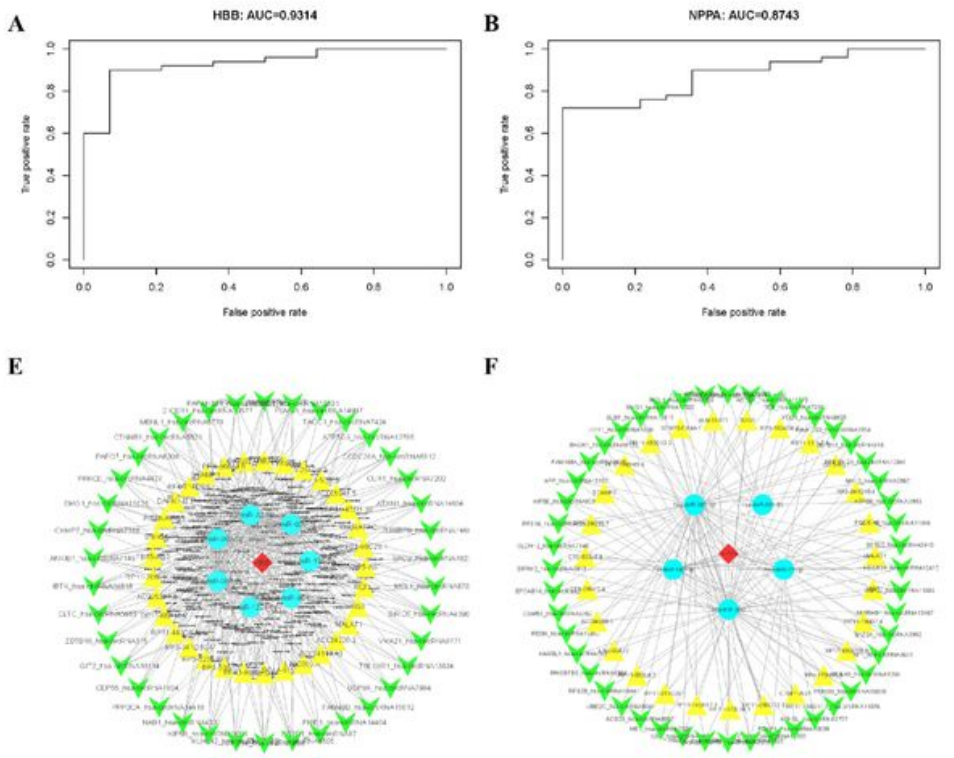

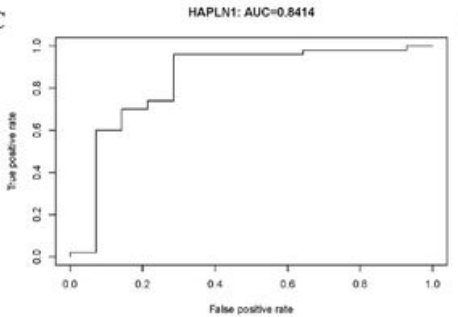

G
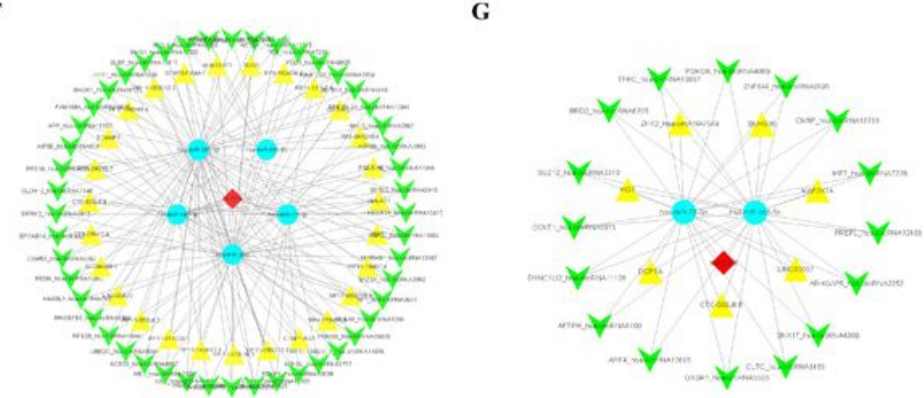

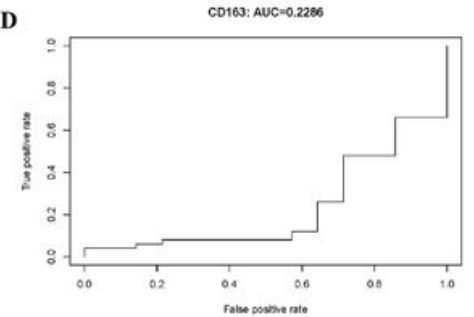

H

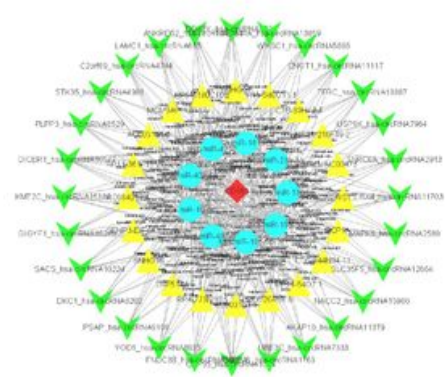

Figure 4

Diagnostic efficacy analysis of hub gene in GSE116250 and ceRNA net-work constrcution. Note: A: ROC diagnosis of HBB gene; $\mathrm{B}$ : ROC diagnosis of NPPA gene; C: ROC diagnosis of HAPLN1 gene; D: ROC 
diagnosis of CD163 gene; E-H: ceRNA network analysis of HBB, NPPA, HAPLN1 and CD163.
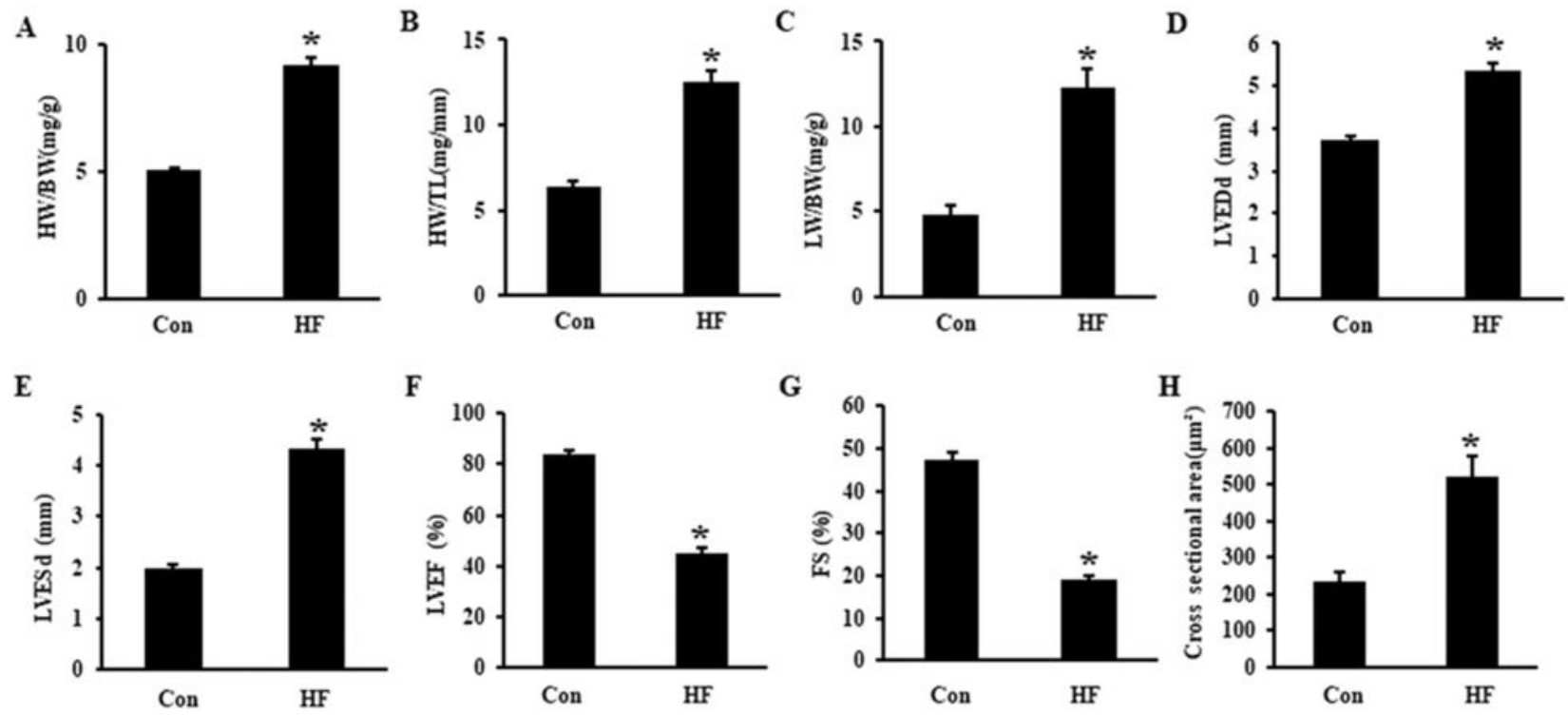

I

J

K
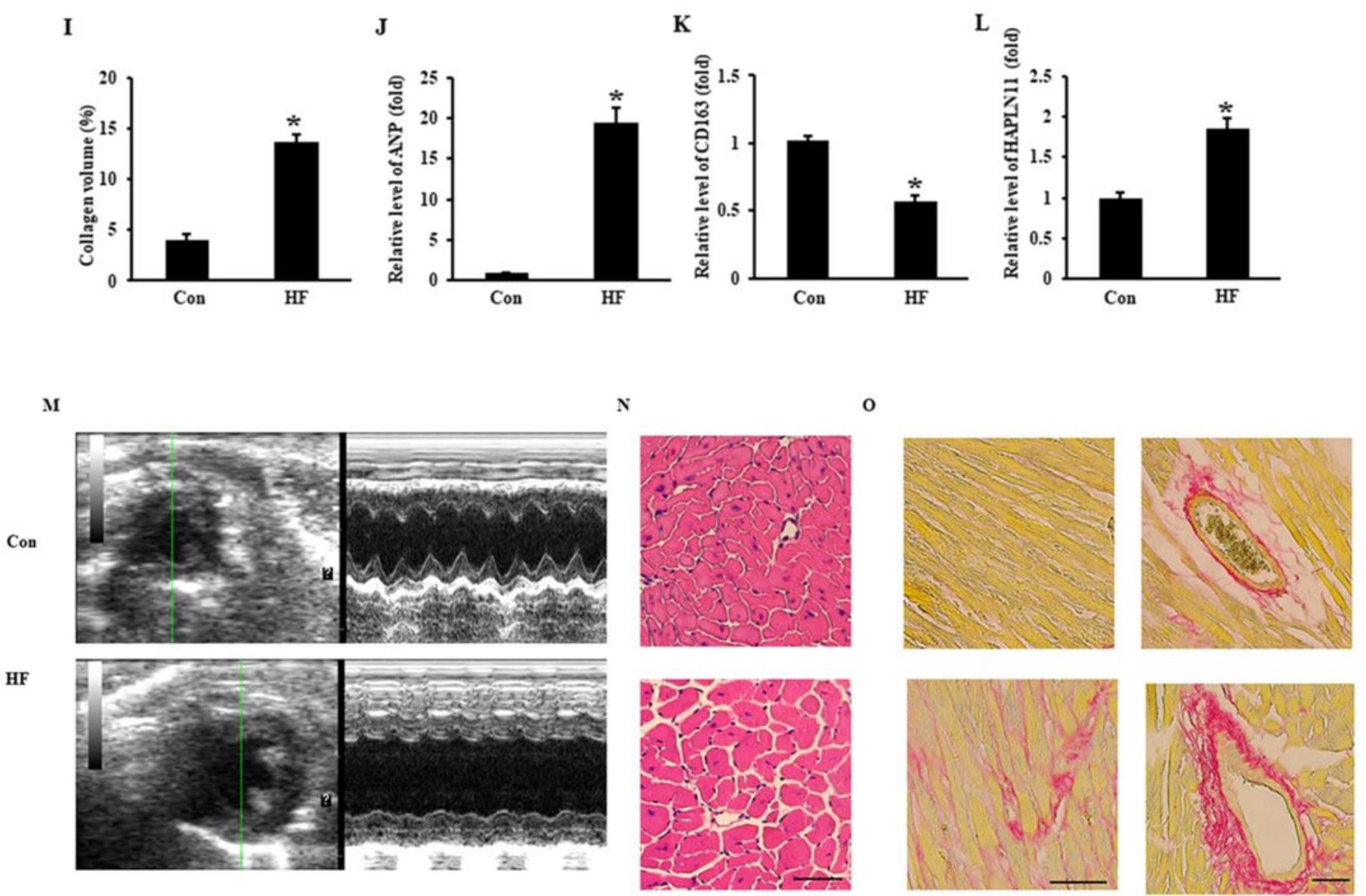

Figure 5

Construction of pressure-overload induced cardiac failure and target gene validation. 
(A-C)-Statistical results of the HW/BW, LW/BW, and HW/TL ratios; (D-G,M)- Echocardiography parameters of LVESd, LVEdd, EF and FS). (H and N) Representative hematoxylin and eosin staining and statistical results for the CSA; (J-L) Reverse transcription-quantitative polymerase chain reaction analysis validation of mRNA expression levels of ANP CD163 and HAPLN1; M-N-H, EF, and FS). (I and O) Representative picrosirius red staining on histological sections and statistical results. ${ }^{*} P<0.05$; The scale bar represents 50um. HW, heart weight; BW, body weight; LW, lung weight; TL, tibial length; LVESd, left ventricular (LV) end-systolic diameter; LVEDd, LV end-diastolic dimension; EF, LV ejection fraction; FS, LV fractional shortening; ANP, atrial natriuretic peptide. 